FTUV/97-65

IFIC/97-95

UALG/TP/97-2

\title{
Remarks on the Reduced Phase Space of $(2+1)$-Dimensional Gravity on a Torus in the Ashtekar Formulation
}

\author{
A. Miković1 ${ }^{\text {f }}$ and N. Manojlović \\ 1. Departamento de Física Teórica and IFIC, Centro Mixto Universidad de Valencia- \\ CSIC, Universidad de Valencia, Burjassot-46100, Valencia, Spain and Institute of \\ Physics, P.O. Box 57, 11001 Belgrade, Yugoslavia \\ 2. Unidade de Ciencias Exactas e Humanas, Sector de Matematica, Universidade do \\ Algarve, Campus de Gambelas, 8000 Faro, Portugal.
}

\begin{abstract}
We examine the reduced phase space of the Barbero-Varadarajan solutions of the Ashtekar formulation of $(2+1)$-dimensional general relativity on a torus. We show that it is a finite-dimensional space due to existence of an infinite dimensional residual gauge invariance which reduces the infinite-dimensional space of solutions to a finite-dimensional space of gauge-inequivalent solutions. This is in agreement with general arguments which imply that the number of physical degrees of freedom for $(2+1)$-dimensional Ashtekar gravity on a torus is finite.
\end{abstract}

\footnotetext{
* E-mail address: mikovic@lie.ific.uv.es

${ }^{\dagger}$ E-mail address: nmanoj@ualg.pt
} 


\section{Introduction}

Ashtekar formlation of General Relativity (GR) [1] takes the spatial connection as the basic dynamical variable, and this has been very fruitfull idea, especially for the formulation of a consistent quantum theory of GR. The Ashtekar formulation can be also applied to $(2+1)$-dimensional GR [2] . $(2+1)$-dimensional GR is a very useful toy model of quantum gravity [3], since it boils down to quantum mechanics while it has enough structure so that various conceptual problems of quantum gravity can be examined. The main reason for this is that the reduced phase space (rps) for $(2+1)$-dimensional GR in the metric formulation is of finite dimension. This also happens in the Witten formulation of $(2+1)$-dimensional GR, which is an alternative connection formulation [4].

Since in the connection formulations the metric is a derived quantity, the phase space contains points corresponding to the degenerate spatial metric. One can show that the Ashtekar formulation is equivalent to the Witten formulation for non-degenerate metrics [2], but in the degenerate sector they are non-equivalent [5]. On the basis of general arguments, one expects that the total reduced phase space of the Ashtekar formulation is also a finite

dimensional symplectic manifold, or a finite union of the former (see [5] for the case of toroidal spatial section). However, Barbero and Varadarajan (BV) have made a claim that the rps for torus is infinite-dimensional [6]. This is done by considering special connection configurations which contain the degenerate metric sectors, and then solving the constraints. In this way one obtains solutions which depend on arbitrary many parameters. Since these parameters are integrals of motion, one concludes that the total rps is infinite-dimensional. This is in contrast to the result of [5], where it was argued that the total rps is of finite dimension.

In this paper we show that every BV solution can be related by a gauge transformation to the solution with a constant connection. This means that BV parameters are not gauge invariant objects or observables, although they 
are integrals of motion. This follows from the fact that BV parameters are Wilson loops, which are not observables in the Ashtekar formulation, although they can be integrals of motion in gauges where the connection is flat. This is also reflected by the fact that the BV gauge for the connection has an infinite-dimensional residual gauge invariance which corresponds to the fact that the gauge is used where the constraints become linearly dependent. Consequently, one can gauge-fix further, so that the spatial coordinate dependence of the corresponding degrees of freedom (dof) is removed. Hence one is left with only one gauge inequivalent dof.

In section two we give a general argument why the rps for $(2+1)$-dimensional Astekar gravity on a torus is of finite dimension, while in section three we explicitely demonstrate our claims about the BV solutions. In section four we present our conclussions.

\section{General considerations}

It is well known that for a gauge theory with $m$ independent gauge symmetries, the number of physical dof is given by $n-m$ fields per spatial point plus $n-m$ corresponding canonicaly conjugate momenta, where $n$ is the number of dynamical components of the gauge field. In the case when $m=n$, there are no local dof, since one can choose a gauge where $q^{i}(x)$ are independent of the spatial coordinates $x$, or more generally, depend on a finite number of constant parameters. The formal argument for this statement is the following. Let $G_{i}(p, q)$ be the first-class irreducible constraints on the phase space $\left(p_{i}(x), q^{i}(x)\right), i=1, \ldots, n$. Solve the constraints $G_{i}=0$ for the momenta $p_{i}$ as $p_{i}=f_{i}\left(q, C_{\alpha}\right)$, where $C_{\alpha}$ are constants of integration, and define

$$
P_{i}(x)=p_{i}(x)-f_{i}\left(q(x), C_{\alpha}\right)
$$

The new variables $P_{i}$ satisfy $\left\{P_{i}, P_{j}\right\}=0$ [7, 8], and $P_{i}=0$ is equivalent to $G_{i}=0$. We can consider $P_{i}$ as the new momenta, and therefore introduce $Q^{i}$ 
as the corresponding coordinates 9]. Therefore the canonical transformation $(p, q) \rightarrow(P, Q)$ makes the constraints abelian. The corresponding gauge invariance also becomes abelian, and it is given by

$$
\delta P_{i}(x)=0 \quad, \quad \delta Q^{i}(x)=\epsilon^{i}(x) \quad .
$$

By using (2) one can set each $Q^{i}$ to zero, and inequivalent solutions will be labeled by $C_{\alpha}$. These are the rps coordinates, and for reparametrization invariant theories $C_{\alpha}$ are observables and hence integrals of motion. What is less clear from (1), is that there are finitely many $C_{\alpha}$. Proving this in the general case may be difficult, but for a concrete theory, one can demonstrate this by showing that a gauge

$$
q^{i}(x)=F^{i}\left(Q_{I}, x\right)
$$

where $\left\{Q_{I}\right\}$ is a finite set of global ( $x$-independent) coordinates, can be allways choosen by performing a gauge transformation on an arbitrary configuration $q^{i}(x)$. In the case of $(2+1)$-dimensional Ashtekar gravity on a torus, this was demonstrated in [5], although not explicitely, so that the dynamics boils down to a constrained particle system.

The explicit argument behind the ansatz from [5] is the following. The Ashtekar constraints for $(2+1)$-dimensional GR can be written as

$$
\begin{aligned}
G_{a} & =D_{i} E^{i}{ }_{a}=\partial_{i} E_{a}^{i}+\epsilon_{a b c} A_{i}{ }^{b} E^{i c}, \\
G_{i} & =F_{i j}{ }^{a} E^{i}{ }_{a} \\
G_{0} & =F_{i j}{ }^{a} E^{i b} E^{j c} \epsilon_{a b c}
\end{aligned}
$$

where $A$ is an $S O(1,2)$ connection one-form on a spatial section $\Sigma, F$ is the corresponding curvature two-form $\left(F_{a}=d A_{a}+\epsilon_{a}{ }^{b c} A_{b} \wedge A_{c}\right)$ and $E$ is the canonicaly conjugate vector density, such that $g^{i j}=E^{i a} E^{j}{ }_{a}$ is a metric density on $\Sigma$. In the case when $\Sigma$ is a torus, one can introduce new phase space variables $\left(\mathcal{A}_{\alpha}{ }^{a}(x), \mathcal{E}^{\alpha}{ }_{a}(x)\right), \alpha=1,2$, via

$$
A_{i}{ }^{a}(x)=\mathcal{A}_{\alpha}{ }^{a}(x) \chi^{\alpha}{ }_{i}(x) \quad, \quad E^{i}{ }_{a}(x)=\mathcal{E}_{a}{ }^{\alpha}(x) L_{\alpha}{ }^{i}(x),
$$


where $\chi^{\alpha}$ are globally defined one-forms, and $L_{\alpha}$ are the corresponding vector fields, which satisfy

$$
d \chi^{\alpha}=0 \quad, \quad \chi_{i}^{\alpha} L_{\beta}^{i}=\delta_{\alpha}{ }^{\beta} \quad, \quad \oint_{\gamma_{\alpha}} \chi^{\beta}=\delta_{\alpha}{ }^{\beta},
$$

where $\gamma_{\alpha}$ are the homology basis curves. The new variables are adapted to the global geometry of the torus. By inserting (7) into (田6) one obtains an equivalent system of six constraints for six configuration variables per space point. Then via (11) and (2), one can set each $\mathcal{A}(x)$ to a constant.

Alternatively, one can show that there are finitely many $C_{\alpha}$ by assuming the opposite, which would mean that there is a local physical dof, since in that case one could construct $C(y)=\sum_{\alpha} C_{\alpha} u_{\alpha}(y)$, where $u_{\alpha}(y)$ is a basis for functions on a sub-manifold of $\Sigma$. This ammounts to introducing a coordinate dependence in $Q_{I}$ from (3), which is the case for the BV solutions. In that case one should show that there is an infinite-dimensional local residual gauge invariance for the gauge choice (3), which reduces the number of dof to a finite number. Both approaches will be employed in the case of the BV solution.

\section{BV solution}

Consider the following one-Killing vector reduction ansatz of the Ashtekar phase-space variables [6]

$$
\begin{aligned}
& E^{\theta}{ }_{a}=E_{1}(\theta) x_{a}, \quad A_{\theta}{ }^{a}=A_{1}(\theta) x^{a} \\
& E_{a}^{\phi}=E_{2}(\theta) y_{a}+E_{3}(\theta) t_{a} \quad, \quad A_{\phi}^{a}=A_{2}(\theta) y^{a}+A_{3}(\theta) t^{a}
\end{aligned}
$$

where $(\theta, \phi)$ are torus coordinates, and $x$ and $y$ are spacelike vectors, while $t$ is a timelike vector, forming a basis in the Lie algebra $s o(1,2) \approx s l(2, R)$. We take a basis $J_{a}, a=1,2,3$, in the fundamental representation of $s l_{2}$ algebra so that $\left[J_{a}, J_{b}\right]=\epsilon_{a b}{ }^{c} J_{c}$ where $\epsilon_{123}=1$ and $\eta_{a b}=2 \operatorname{tr}\left(J_{a} J_{b}\right)=\operatorname{diag}(1,1,-1)$. Therefore $x=J_{1}, y=J_{2}$ and $t=J_{3}$. By inserting the ansatz (9-10) into 
(46) we obtain the following constraints

$$
\begin{aligned}
& G_{1}=E_{1}^{\prime}+A_{2} E_{3}-A_{3} E_{2}=0, \\
& G_{2}=E_{2} f_{2}-E_{3} f_{3}=0, \\
& G_{3}=E_{1}\left(E_{2} f_{3}-E_{3} f_{2}\right)=0,
\end{aligned}
$$

where $f_{2}=A_{2}{ }^{\prime}-A_{1} A_{3}$ and $f_{3}=A_{3}{ }^{\prime}-A_{1} A_{2}$ are the non-zero components of $F$ and $I=d / d \theta$. The corresponding dynamical system is defined by the action

$$
S=\int d t \int_{0}^{2 \pi} d \theta\left(E_{i} \dot{A}_{i}-\lambda^{i} G_{i}\right),
$$

where $\lambda^{i}$ are the Lagrange multipliers enforcing the constraints (11-13). The constraints generate the gauge invariance of the action

$$
\begin{aligned}
& \delta A_{i}=\int_{0}^{2 \pi} d \theta\left\{\epsilon^{j} G_{j}, A_{i}\right\} \quad, \quad \delta E_{i}=\int_{0}^{2 \pi} d \theta\left\{\epsilon^{j} G_{j}, E_{i}\right\} \\
& \delta \lambda^{i}=\dot{\epsilon^{i}}+\int_{0}^{2 \pi} \int_{0}^{2 \pi} d \theta_{1} d \theta_{2} \lambda^{j}\left(\theta_{1}\right) \epsilon^{k}\left(\theta_{2}\right) f_{j k}^{i}\left(\theta_{1}, \theta_{2}, \theta\right)
\end{aligned}
$$

where $f$ are the structutre functions of the constraint algebra. The explicit gauge transformations for the phase-space variables are given by

$$
\begin{aligned}
& \delta A_{1}=-\frac{d \epsilon^{1}}{d \theta}+\left(E_{2} f_{2}-E_{3} f_{3}\right) \epsilon^{3} \\
& \delta A_{2}=-A_{3} \epsilon^{1}+f_{2} \epsilon^{2}+E_{1} f_{3} \epsilon^{3} \\
& \delta A_{3}=A_{2} \epsilon^{1}-f_{3} \epsilon^{2}-E_{1} f_{2} \epsilon^{3}
\end{aligned}
$$

and

$$
\begin{aligned}
& \delta E_{1}=\left(E_{2} A_{3}-E_{3} A_{2}\right) \epsilon^{2}+\left(E_{2} A_{2}-E_{3} A_{3}\right) E_{1} \epsilon^{3} \\
& \delta E_{2}=-E_{3} \epsilon^{1}+\left(E_{2} \epsilon^{2}\right)^{\prime}-A_{1} E_{3} \epsilon^{2}-\left(E_{1} E_{3} \epsilon^{3}\right)^{\prime}+A_{1} E_{1} E_{2} \epsilon^{3} \\
& \delta E_{3}=E_{2} \epsilon^{1}-\left(E_{3} \epsilon^{2}\right)^{\prime}+A_{1} E_{2} \epsilon^{2}+\left(E_{1} E_{2} \epsilon^{3}\right)^{\prime}-A_{1} E_{1} E_{3} \epsilon^{3} .
\end{aligned}
$$

The equations of motion can be obtained from $(17-22)$ by replacing the variations with the time derivatives and the gauge parametars with the lagrange multipliers. 
An immediate indication that the dynamical system (14) will not posses local dof is the fact that there are 3 functionally independent constraints acting on 3 configuration variables per space point. This can be demonstrated in the following way. Let us choose a gauge for the connection such that $F$ is non-null, i.e. $f_{2}^{2}-f_{3}{ }^{2} \neq 0$. Then the constraints imply

$$
E_{1}^{\prime}=E_{2}=E_{3}=0 .
$$

Now let us choose a gauge where $F$ is null, i.e. $f_{2}= \pm f_{3}$. Then the constarints give

$$
E_{1}{ }^{\prime}=\left(A_{3} \mp A_{2}\right) E_{2} \quad, \quad E_{2}= \pm E_{3} \quad,
$$

where $E_{2}$ is arbitrary. It looks like the configurations (24) have a local dof. However, by performing a gauge transformation on a null connection, one can always reach a non-null connection, since

$$
\delta\left(f_{2} \mp f_{3}\right)=\left[2 \epsilon-\left(A_{3} \pm A_{2}\right) \epsilon^{1}\right]^{\prime} \pm A_{1}\left[2 \epsilon-\left(A_{3} \pm A_{2}\right) \epsilon^{1}\right] \pm\left(A_{3}-A_{2}\right) d \epsilon^{1} / d \theta,
$$

where $\epsilon= \pm f_{2}\left(\epsilon^{2} \pm E_{1} \epsilon^{3}\right)$, so that one can always choose the $\epsilon$ 's such that the left hand side becomes non-zero. Therefore the configuration (24) is gauge equivalent to (23). The same applies to the $f_{2}=f_{3}=0$ configuration. Hence the gauge inequivalent solutions are labeled by (23), which in turn are labeled by $E_{1}=e_{1}=$ const. and $A_{1}=a_{1}=$ const. canonical pair. This corresponds to the fact that

$$
a_{1}=\int_{0}^{2 \pi} d \theta A_{1}
$$

is the true integral of motion, or the observable, since its Poisson bracket with the constraints is weakly zero.

In the case of the BV solution the following gauge is fixed for the connection

$$
A_{1}=A_{2}=A \quad, \quad A_{3}=A+c_{0} \exp \left(-\int_{0}^{\theta} d \theta A\right) \quad, \quad c_{0} \neq 0,
$$

where $A$ is an arbitrary smooth function of $\theta$, such that $A \neq 0$ in $\left(a_{k}, b_{k}\right)$, where $0<a_{1}<b_{1}<\ldots<a_{n}<b_{n}<2 \pi$. The intervals $\left(a_{k}, b_{k}\right)$ are called 
null patches, since the conection curvature vector $F^{a}=\epsilon^{i j} F_{i j}{ }^{a}$ is null there $\left(f_{2}=f_{3}\right)$. In that case the constraints give

$$
E_{3}=E_{2} \quad, \quad E_{1}^{\prime}=\left(A_{3}-A_{2}\right) E_{2}
$$

This solution belongs to the degenerate metrics sector, since

$$
\operatorname{det}\left\|g^{i j}\right\|=E_{1}^{2}\left(E_{2}^{2}-E_{3}^{2}\right)=0 \text {. }
$$

In the flat patches $\left(b_{k}, a_{k+1}\right), A=0$ and $F=0$, so that the solution is given by

$$
A_{3}=c_{k} \quad, \quad E_{1}{ }^{\prime}=A_{3} E_{2} \quad, \quad E_{2}=1 / c_{k} \quad, \quad E_{3} \neq E_{2},
$$

where $c_{k}=A_{3}\left(b_{k}\right)$, and $E_{3}$ is an arbitrary function satisfying the boundary conditions. The last condition in (30) insures that the metric is nondegenerate, so that the trace of a holonomy of a loop (Wilson loop) in a flat patch

$$
W=\operatorname{tr} U=\operatorname{tr} P \exp \left(\int_{0}^{2 \pi} A_{\phi} d \phi\right)
$$

is an integral of motion given by $2 \cos \left(\pi c_{k}\right)$. Since the solution in the null patches (28) depends on the unconstrained canonical pair $\left(A_{2}, E_{2}\right)$, it follows that one can specify points in the part of the reduced configuration space by arbitrary many independent parameters $\left(c_{1}, \cdots, c_{n}\right)$, and hence one concludes that the dimension of the rps cannot be finite.

This would be true provided that the null-patch solutions (28) with different functions $A$ are gauge inequivalent. However, this is not the case, which follows from the fact that the function $A$ must satisfy

$$
\int_{0}^{2 \pi} d \theta A=0
$$

This means that BV solutions belong to the $a_{1}=0$ class, and hence they are all gauge equivalent to $A=0$ solution, for which $c_{1}=\cdots=c_{n}$. Hence the quantities $c_{k}$ are not gauge invariant objects, or observables, although they are integrals of motion. This follows from the fact that $W_{k}=2 \cos \left(\pi c_{k}\right)$ are 
traces of holonomies, and these are not observables in the Ashtekar formulation, although they can be integrals of motion in the gauges where $F=0$ in some parts of the torus, like in the BV case.

One can check that the BV gauge is dynamically consistent, which ammounts to imposing the time preservation of the gauge-fixing functions

$$
F_{1}=A_{1}-A_{2}=0 \quad, \quad F_{2}=\left(A_{3}-A_{1}\right)^{\prime}+A_{1}\left(A_{3}-A_{1}\right)=0 .
$$

From $d F_{1} / d t=0$ and $d F_{2} / d t=0$, the equations of motion imply

$$
-d \lambda^{1} / d \theta+A_{3} \lambda^{1}=f_{2}\left(\lambda^{2}+E_{1} \lambda^{3}\right)
$$

By further requiring that the relations (28) are preserved in time one finds that the Lagrange multipliers are fixed as

$$
\lambda^{1}=0 \quad, \quad \lambda^{2}=-E_{1} \lambda^{3}=-C / 2\left(A_{3}-A_{1}\right)^{-1},
$$

where $C$ is an arbitrary constant. In the flat patches the dynamical consistency requires $\lambda_{1}=0$, so that $\dot{A}_{2}=\dot{A}_{3}=0$ and hence the corresponding Wilson loop is independent of time. However, since the Wilson loop is not an observable in the Ashtekar formulation, one can find a gauge where it will be time dependent. This can be seen from the following derivation. By using a formula

$$
\exp \left(2 \theta_{2} J_{2}+2 \theta_{3} J_{3}\right)=\operatorname{ch} \sqrt{\theta_{2}^{2}-\theta_{3}^{2}}+\frac{\operatorname{sh} \sqrt{\theta_{2}^{2}-\theta_{3}^{2}}}{\sqrt{\theta_{2}^{2}-\theta_{3}^{2}}}\left(2 \theta_{2} J_{2}+2 \theta_{3} J_{3}\right),
$$

the Wilson loop (31) can be evaluated explicitely

$$
W=\operatorname{tr} \exp \left(2 \pi A_{2} J_{2}+2 \pi A_{3} J_{3}\right)=2 \operatorname{ch}\left(\pi \sqrt{A_{2}^{2}-A_{3}^{2}}\right) \quad .
$$

In a flat patch one then has

$$
\dot{W}=-4 \pi \frac{\operatorname{sh}\left(\pi \sqrt{A_{2}^{2}-A_{3}^{2}}\right)}{\sqrt{A_{2}^{2}-A_{3}^{2}}} \lambda^{1} A_{2} A_{3}
$$


from which is clear that $W$ will be time dependent in gauges where $\lambda^{1} A_{2} A_{3} \neq$ 0 . An example of such a gauge is

$$
A_{1}=0 \quad, \quad A_{2}=\alpha(\theta)+d_{k} \quad, \quad A_{3}=\alpha(\theta)+h_{k},
$$

where $d_{k} \neq h_{k}$ are constants, and $\alpha(\theta)$ takes constant values in flat patches, so that $f_{2}=f_{3}=0$, while in null patches $\alpha^{\prime}(\theta) \neq 0$ and hence $f_{2}=f_{3} \neq 0$. The gauge (39) is an alternative realisation of the null and flat patch initial data, and the dynamical consistency requires that $\lambda_{1}=-1$. In a flat patch one then has

$$
\dot{A}_{1}=0 \quad, \quad \dot{A}_{2}=\lambda^{1} A_{3} \quad, \quad \dot{A}_{3}=-\lambda^{1} A_{2},
$$

so that

$$
A_{2}=\alpha_{k} \cos \left(\lambda^{1} t+\varphi_{k}\right) \quad, \quad A_{3}=\alpha_{k} \sin \left(\lambda^{1} t+\varphi_{k}\right)
$$

where $\alpha+d_{k}=\alpha_{k} \cos \varphi_{k}$ and $\alpha+h_{k}=\alpha_{k} \sin \varphi_{k}$. Hence the Wilson loop (31) is not an observable, since it is possible to find gauges where $\dot{W} \neq 0$.

\section{Residual gauge transformations}

Our arguments from the previous section imply that the BV solution with arbitrary $c_{k}$ coefficients is gauge equivalent to $c_{1}=\ldots=c_{n}$ solution. This means that the gauge (33) has an infinite-dimensional residual gauge invariance. One can find this residual gauge invariance from the requirements $\delta F_{1}=\delta F_{2}=0$. They give

$$
-d \epsilon^{1} / d \theta+A_{3} \epsilon^{1}=f_{2}\left(\epsilon^{2}+E_{1} \epsilon^{3}\right)
$$

Equation (42) implies that the gauge parameters $\epsilon^{2}$ and $\epsilon^{3}$ are not fixed, so that the corresponding gauge transformations will preserve the gauge (33). This is a direct consequence of the fact that in the BV gauge the constraints $G_{2}$ and $G_{3}$ become linearly dependent. Note that in a non-null gauge $\left(f_{2} \neq\right.$ 
$\pm f_{3}$ ), where the constraints are independent, the residual transformations for static solutions are given by

$$
\begin{aligned}
& \delta A_{1}=-\frac{d \epsilon^{1}}{d \theta}=0, \\
& \delta A_{2}=-A_{3} \epsilon^{1}+f_{2} \epsilon^{2}+E_{1} f_{3} \epsilon^{3}=0 \\
& \delta A_{3}=A_{2} \epsilon^{1}-f_{3} \epsilon^{2}-E_{1} f_{2} \epsilon^{3}=0
\end{aligned}
$$

These equations completely fix the $\epsilon$ 's, and consequently there is no residual gauge invarince, which corresponds to the fact that in such a gauge the solution contains only the physical degrees of freedom.

This analysis implies that the Wilson loop (31) will not be invariant under the residual gauge transformations in flat patches. This can be seen from the expression (37) since

$$
\delta W=-4 \pi \frac{\operatorname{sh}\left(\pi \sqrt{A_{2}^{2}-A_{3}^{2}}\right)}{\sqrt{A_{2}^{2}-A_{3}^{2}}} \epsilon^{1} A_{2} A_{3} .
$$

In the $\mathrm{BV}$ gauge this variation is zero, since $A_{2}=0$. However, the residual transformations (42) allow one to choose a gauge in a flat patch such that $A_{2} \neq 0$, and hence the variation (46) will be non-zero. Really, the infinitesimal gauge transformations (42) imply that the BV configuration $A_{1}=A_{2}=0$ and $A_{3}=c_{k}$ transforms into

$$
A_{1}=A_{2}=-c_{k} \epsilon^{1}(\theta) \quad, \quad A_{3}=c_{k}
$$

for which the variation (46) is non-zero, and it is of order $\left(\epsilon^{1}\right)^{2}$. This means that the variation (46) will be non-zero at the second order in $\epsilon^{1}$ if one starts from the $A_{2}=0$ configuration. Note that the configuration (47) corresponds to the lowest order terms in the $\epsilon^{1}$ expansion of the configuration

$$
A_{1}=A_{2}=-A_{3}=\frac{1}{\theta-\theta_{k}}
$$

where $\theta_{k}$ is a constant. This follows from the fact that the equations

$$
f_{2}=A^{\prime}-A A_{3}=0 \quad, \quad f_{3}=A_{3}{ }^{\prime}-A^{2}=0,
$$


have (48) as the unique solution for $A \neq 0$. In the configuration (48) one finds that $W(A)=2$, and hence $c_{1}=\ldots=c_{n}=0$. Therefore $W(A) \neq W(\tilde{A})$, where $A$ and $\tilde{A}$ are related by a gauge transformation, which confirms our result from the previous section that the Wilson loop is not an observable, and also shows that the configuration with arbitrary $c_{k}$ 's is gauge equivalent to $c_{k}=0$ configuration.

Therefore the gauge inequivalent BV solutions are labeled with only one parameter $e_{1}$, which is canonicaly conjugate to the gauge invariant parameter $a_{1}$. The corresponding phase space is labeled by $\left(a_{1}, e_{1}\right)$, which is a subspace of the total rps given locally by the four-dimensional symplectic manifold $\mathbf{R}^{4}$ [5].

\section{Conclussions}

We have demonstrated that the number of physical dof for $(2+1)$-dimensional Ashtekar gravity on a torus is finite, which confirms the correctness of the ansatz of [5]. It would be an interesting problem to show that the number of physical dof is finite for a higher-genus $\Sigma$.

It is important to realize that an integral of motion does not have to be an observable, and this is the reason why Barbero and Varadarayan have made a wrong conclussion about the dimensionality of the rps. The more familiar examples of this situation are the static solutions of $2 \mathrm{~d}$ dilaton gravities, which include the spherically symmetric GR solutions. In that case the components of the metric are independent of time, and hence they are integrals of motion. However, the metric is obviously not an observable. The observable is the ADM mass, which labels the gauge inequivalent solutions. In the BV case, the role of the ADM mass is played by $a_{1}$. Also, in analogy

to the gauge (41), one can find gauges in the $2 \mathrm{~d}$ dilaton gravity case where the metric is time dependent.

From the point of view of the full Ashtekar theory, the Wilson loop $W$ can 
be an observable only if $F=0$ and $\operatorname{det} E \neq 0$ conditions can be preserved in time independently of the gauge, or equivalently, of the value of the Lagrange multipliers. However, although the $F=0$ condition can be preserved in time for every initial flat configuration, the metric non-degeneracy condition $\operatorname{det} E \neq 0$ can not be preserved in time for every inital configuration, which explains why in the case of the gauge (39) the Wilson loop becomes time dependent in a flat patch.

Note that the BV solution can be understood from the general theory of gauge fixing (see [10] and references there) in the following way. For a dynamical system with first-class irreducible constraints $G_{\alpha}(p, q)$, the rps is obtained by choosing the gauge fixing conditions $\chi^{\alpha}(p, q)=0$ such that

$$
\left\{\chi^{\alpha}, \chi^{\beta}\right\}=0
$$

and the Faddeev-Popov determinat

$$
\Delta=\left.\operatorname{det}\left\{G_{\alpha}, \chi^{\beta}\right\}\right|_{\chi=0}
$$

must be different from zero. In the infinite-dimensional case, $\Delta$ has to be carefully defined, because of the presence of the trivial zero modes, which should be omitted. Therefore the condition of non-zero $\Delta$ in the infinite dimensional case means that the operator $\hat{\Delta}$, defined by $\left.\left\{G\left(x_{1}\right), \chi\left(x_{2}\right)\right\}\right|_{\chi=0}$, must not have non-trivial zero eigenvalues. This translates into examining the solutions of the equation $(\alpha=\{i, x\})$

$$
\hat{\Delta}^{i}{ }_{j} \epsilon^{j}(x)=\left.\int d x_{1} \epsilon^{j}\left(x_{1}\right)\left\{G_{j}\left(x_{1}\right), \chi^{i}(x)\right\}\right|_{\chi=0}=0 .
$$

But this is precisely the condition for finding the residual gauge invariances. Finding precise criteria for a non-trivial solution depends on a concrete theory, but it is clear that a solution for which one or more of $\epsilon^{i}(x)$ are unrestricted is non-trivial. Such solutions appear in the gauge (27), which means

\footnotetext{
${ }^{\ddagger}$ In the case of a reparametrization invariant system, this condition must be generalized to $\chi^{\alpha}(p, q)=\delta_{1}{ }^{\alpha} f(t)$, where $t$ is the evolution parameter [10]. This amounts to choosing the time variable in the system. The gauge choice (27) should be modified accordingly, but this does not affect the subsequent analysis.
} 
that a gauge is chosen such that the equations $\left.G_{i}\right|_{\chi=0}=0$ are linearly dependent. In other words, when solving the constraints in such gauges, one uses only a part of the constraints, and that is the reason why one obtains the solutions with more dof than the number of physical dof.

\section{References}

[1] A. Ashtekar, Phys. Rev. D 36 (1987) 1587

[2] I. Bengtsson, Phys. Lett. B 220 (1989) 51

[3] S. Carlip, Lectures on (2+1)-dimensional gravity (1995), gr-qc/9503024

[4] E. Witten, Nucl. Phys. B 311 (1988) 46

[5] N. Manojlović and A. Miković, Nucl. Phys. B 385 (1992) 571

[6] J.F. Barbero and M. Varadarajan, Nucl. Phys. B 415 (1995) 515

[7] M. Henneaux, Phys. Rept. 126 (1985) 1

[8] K. Kuchar, in Quantum Gravity 2: A Second Oxford Symposium, eds C.J. Isham et al (Clarendon, Oxford, 1981)

[9] L.D. Faddeev, Theor. Math. Phys. 1 (1970) 1

[10] N. Manojlović and A. Miković, Nucl. Phys. B 382 (1992) 148 